ARTICLE

https://doi.org/10.1038/s41467-020-17361-8

\title{
Rapid and high-resolution patterning of microstructure and composition in organic semiconductors using 'molecular gates'
}

\author{
Aleksandr Perevedentsev (iD ${ }^{1 凶} \&$ Mariano Campoy-Quiles (iD ${ }^{1 凶}$
}

\begin{abstract}
Photolithography has been a major enabling tool for miniaturisation of silicon devices that underpinned the electronics revolution. Rapid, high-resolution patterning of key material characteristics would, similarly, accelerate the advent of molecular electronics and photonics. Here we advance a versatile approach employing local diffusion of functional small-molecular compounds through a solution-processed 'molecular gate' interlayer. Diffusion is activated using laser light or solvent vapour jets_ _ a process that can be finely modulated down to molecule-on-demand deposition precision with almost photolithographic resolution $(<5 \mu \mathrm{m})$ and speeds $\left(3 \mathrm{~mm} \mathrm{~s}^{-1}\right)$. Examples of principal pattern types are presented including molecular conformation for integrated photonics; chain orientation for polarised security features and micro-engineered electronics; and doping with local conductivity values $>3 \mathrm{~S} \mathrm{~cm}^{-1}$ for improved electronic devices. Finally, we demonstrate the unique capability for one-step patterning of multiple functionalities by spatially modulating composition in ternary blends, leading to locally tunable photoluminescence from blue to red.
\end{abstract}

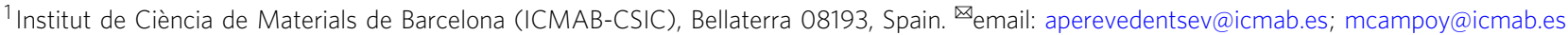


W e hold the fact to be self-evident that the field of molecular electronics and photonics ${ }^{1-3}$ is reaching its promise, with several classes of high-performance, lightweight and flexible devices appearing on the market or nearing commercialisation. Besides potentially novel functionalities that may complement, or go beyond, those of inorganic semiconductors, two of the principal motivations underlying the growth of this field are, arguably, economic and environmental, given the cost and energy efficiency improvements that can be achieved by large-area low-temperature solution-based fabrication.

A key part of fabrication of organic semiconductor active layers involves spatial patterning of material characteristics to enable device-specific functionalities ${ }^{4-6}$. Of these, the principal characteristic is material composition, as required for fabrication of, e.g., interconnects and electrodes for organic field-effect transistors by local doping ${ }^{7}$ and emissive components for organic lightemitting diodes (OLEDs) ${ }^{4}$. For polymeric semiconductors, two additional microstructural characteristics are of practical importance, namely (i) chain conformation, which affects refractive index ${ }^{8}$ and other optoelectronic properties ${ }^{9,10}$, and (ii) chain orientation, which enables anisotropic electronic ${ }^{11,12}$ and ther$\mathrm{mal}^{13}$ conductivities, as well as polarised absorption and emission of light ${ }^{14,15}$.

Numerous approaches towards patterning organic semiconductors have been reported; excellent reviews can be found in refs. ${ }^{4,6}$. These can be broadly classified into light-based methods such as photolithography 16 and laser-induced forward transfer (LIFT) ${ }^{17,18}$; printing methods such as inkjet, vapour jet and aerosol jet ${ }^{19-23}$, and contact methods such as hard and soft imprint lithography ${ }^{24-26}$. Of these, photolithography represents the most mature technology, with a further advantage being its high spatial resolution $(\sim 1 \mu \mathrm{m}$ in large-field proximity-mode implementations). Inkjet printing, on the other hand, offers superior versatility and speed, albeit with lower resolution (typically $\sim 30-50 \mu \mathrm{m}$ ).

The availability of this plurality of patterning techniques is promisingly moving the field forwards, with an increasing number of reports on devices with integrated components. Nevertheless, particularly in the context of optimal large-area, roll-to-roll-type processing, the aforementioned techniques are subject to a number of limitations. First, the available techniques typically are additive or subtractive, requiring an additional backfilling step to return to a planar film format. Second, many of the patterning strategies necessitate slow, multi-step processing as in the case of photolithographic approaches. Third, several structural features remain generally unattainable, notably local patterning of molecular orientation, which is either limited to specific compounds ${ }^{27}$, demands the restrictive use of insulating alignment layers ${ }^{28}$ or requires very specific geometries ${ }^{29,30}$. Fourth, most patterning approaches are 'binary', with a single structural feature defined on the baseline layer in one patterning step, thus requiring additional time-consuming post-processing or mask alignment in cases when multiple patterns or functionalities are desired.

Recognising these limitations of the state-of-the-art, we advance a conceptually different approach to micro-patterning of organic semiconductors based on donor diffusion through a 'molecular-gate' interlayer, which provides both the spatial resolution of photolithography and the versatility of printing techniques. By selection of appropriate donor compounds, we demonstrate spatial patterning of chain conformation and orientation, as well as material composition, in several benchmark semiconducting polymers. Given the use of vacuum-free solutionbased processing and non-contact stimuli such as laser light, the reported patterning approach is expected to be highly suited for rapid micro-patterning of advanced device structures in both rollto-roll and laboratory-scale environments.

\section{Results}

The 'molecular-gate' concept. The concept underlying the patterning method advanced herein is the controlled, stimulusinduced diffusion of functional small molecules into the target film through a permeation-switchable membrane. As illustrated below and in Supplementary Fig. 1, the method employs the following sequential processing steps (the terminology will be adopted hereafter): first, solution-based deposition of a target semiconductor film, a 'molecular-gate' interlayer and a donor layer, which comprises functional small-molecular compound(s). This is followed by application of thermal-, solvent-vapour- or laser-based stimulus to activate diffusion of the functional compound(s) of the donor layer into the target film through the molecular gate. The residual donor layer and molecular gate are subsequently removed in the final processing step. Supplementary Movie 1 shows an animated illustration of the molecular-gate concept and exemplary applications thereof.

The molecular-gate functions as a semipermeable membrane, preventing uncontrolled diffusion of functional compounds into the target film (e.g., during solution-based deposition of donor layer) and allowing diffusion under an external stimulus such as heat or solvent vapour. Using diffusion through a $\sim 100 \mathrm{~nm}$ interlayer as the physical mechanism for patterning _ rather than droplet transfer as in inkjet printing or LIFT concept$s^{17}$ _ enables essentially a molecule-by-molecule deposition, similar to the thermal evaporation process but with high spatial resolution, mask-free and no vacuum requirements.

The desired requirements for the molecular-gate material include preferential solubility in solvents orthogonal to those used for depositing typical organic semiconductors and donor compounds, high molecular weight to ensure good filmforming ability, and high thermal stability for minimal interdiffusion with adjacent layers during patterning. Hence, poly (sodium 4-styrenesulfonate) ( $\mathrm{pNaSS}$ ) was selected, given that it features glass transition and decomposition temperatures of $211^{\circ}$ $\mathrm{C}$ and $>470^{\circ} \mathrm{C}$, respectively 31,32 , with high-molecular-weight materials commercially available at a moderate cost. Its solubility in water and insolubility in common organic solvents allow for its straightforward deposition onto organic semiconductor films and post-patterning removal as well.

Drawing inspiration from our previous work 8,33 , here a macroscopic proof-of-concept demonstration of molecular-gatebased patterning is provided using chain-conformation-mediated photoluminescence (PL) switching in poly(9,9-dioctylfluorene) (PFO) films. PFO exhibits a well-known conformational isomer, termed the ' $\beta$-phase', corresponding to an extended, planarzigzag geometry, which thus features a distinctly red-shifted PL spectrum relative to an in-plane-isotropic, glassy sample (PL peaks at $\sim 438$ and $423 \mathrm{~nm}$, respectively) ${ }^{9}$. $\beta$-Phase formation is induced in glassy PFO by exposure to various small-molecular 'solvents', proceeding via a co-crystallisation process ${ }^{34,35}$. The overall PL is extremely sensitive to the presence of even a small, $\leq 1 \%$ fraction of $\beta$-phase chain segments ${ }^{9}$, offering an ideal material system for demonstrating the robustness of the proposed patterning approach, as well as the molecule-on-demand concept.

Figure 1 illustrates the individual processing steps. The baseline glassy PFO film is spin-coated on a glass substrate (step 1), exhibiting dark-blue PL under ultraviolet (UV) illumination. Subsequent spin-coating of pNaSS from solutions in water (step 2) yields a homogeneous film without altering the PL characteristics of PFO. The presence of the pNaSS molecular gate protects the PFO film during the following spin-coating of lauric acid (LA) 
a
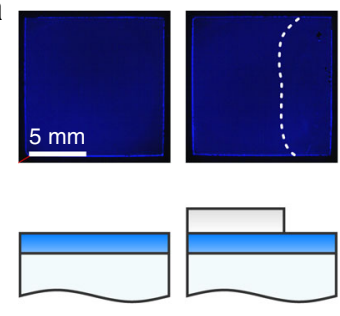

b
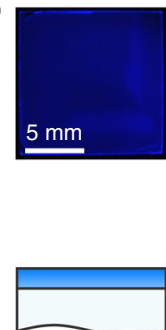
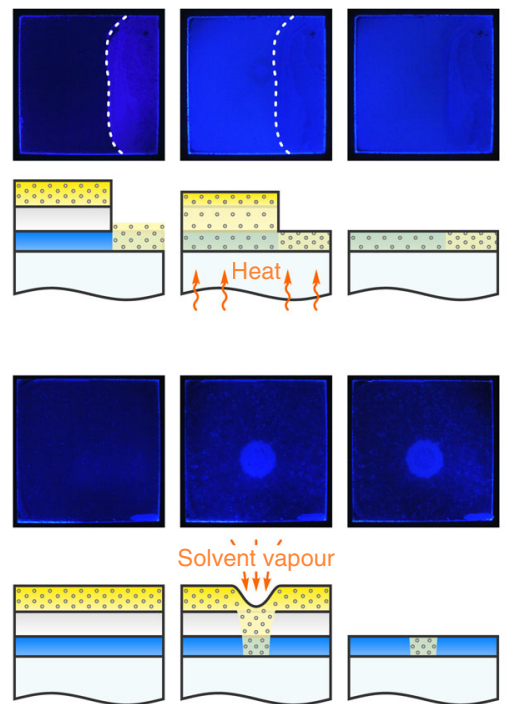
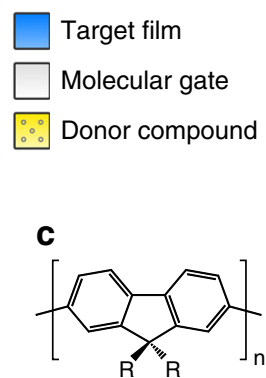

d

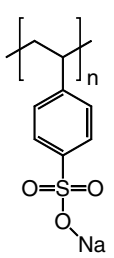

Fig. 1 Illustration of the molecular-gate concept by PL switching in PFO films. Examples are shown for (a) thermal and (b) solvent-vapour-based stimuli. Top rows show photographs of the films under UV light illumination at the sequential processing steps, depicted schematically in the bottom rows. These are: deposition from solutions of the target film (PFO; depicted in blue), the 'molecular gate' (pNaSS; depicted in grey) and the functional donor compound (lauric acid; depicted in yellow + grey circles); followed by application of heat or solvent vapour to diffuse lauric acid into the PFO film via the molecular gate and, finally, removal of the residual donor layer and gate by spin-off. In the second step of (a), a part of the gate was removed by immersion in water (as shown by the dotted line) to highlight its role in preserving the 'baseline' characteristics of the target film during donor layer deposition. Also shown are the chemical structures of (c) PFO $\left(R=\mathrm{C}_{8} \mathrm{H}_{17}\right)$ and (d) pNaSS.

(step 3) - the functional 'solid solvent' employed for inducing the conformational transformation. It is noteworthy that depositing LA directly onto PFO (step 3 in Fig. 1a) immediately induces the $\beta$-phase, as evidenced by the appearance of light-blue PL, thereby preventing any opportunities for local patterning.

Controlled diffusion of LA (step 4) into the PFO film is activated by heating slightly above the melting temperature of LA $\left(T_{\mathrm{m}}=44^{\circ} \mathrm{C}\right.$; Fig. 1a) or exposure to solvent vapour (Fig. $1 \mathrm{~b}$ ) that renders the small-molecule mobile, resulting in formation of the $\beta$-phase and the corresponding change in PL. Exposure to solvent vapour through a nozzle ${ }^{33}$ (step 4 in Fig. 1b) enables a local change in the chain conformation through the interaction of LA with the polymer (note that acetone and water are non-solvents of PFO and, in the absence of LA, do not induce $\beta$-phase formation). The residual LA donor layer and $\mathrm{pNaSS}$ gate are finally removed by spin-off (step 5) with solvents such as acetone and water (orthogonal to PFO and the majority of other organic semiconductors) without dissolving, or further modifying, the PFO film. Reassuringly, the PL emission colour is found to be very homogeneous for the sample area where LA diffused through the gate-and less so for the area where LA was deposited onto PFO directly (cf. left and right sides of panel (5) in Fig. 1a)-indicating that gated diffusion is a highly controllable process.

Key to realisation of patterning, therefore, are the selection of small-molecular compounds with the desired functionality, as well as delivering a local stimulus for 'opening' the gate and enabling spatially selective diffusion. The stimulus, whether heator solvent-vapour-based, is employed to impart molecular mobility to the compounds comprising the donor layer by melting or swelling. The resulting rate of diffusion of said compounds into the donor layer scales exponentially with temperature and is governed by additional parameters such as glass transition of the target semiconductor film, molar mass of the functional compounds and thickness of the molecular gate ${ }^{36}$. As laser light is the most versatile choice of stimulus, the following sections will present local patterning of various feature types by laser-induced 'gated' diffusion.

Patterning chain conformation. Laser patterning of the $\beta$-phase chain conformation in PFO films is performed in the configuration shown in Fig. 2a, with a more detailed, step-by-step schematic illustration presented in Supplementary Fig. 1. PFO/ $\mathrm{pNaSS} / \mathrm{LA}$ trilayer samples (as above) are deposited on indium tin oxide (ITO)/glass substrates. Laser excitation at $785 \mathrm{~nm}$ (nonresonant for PFO) is used to generate local heating at the substrate level by partial absorption within ITO, activating diffusion of LA into PFO in an area that is essentially defined by the excitation spot and laser parameters.

PL microscopy images of $\beta$-phase lines patterned by continuous scanning of the sample in the laser focal plane at a constant writing speed $v$ and varying power $P$ are shown in Fig. $2 \mathrm{~b}$, with spectral filtering at $438 \mathrm{~nm}$ used to preferentially select the peak of $\beta$-phase emission ${ }^{8}$. (Full PL spectra are shown in Supplementary Fig. 2a.) As is apparent from $\beta$-phase line widths increasing with $P$, laser power represents a key parameter that modulates pattern features by governing the local temperature rise and, therewith, the diffusivity of the functional small molecule. Writing speed is another parameter that determines the time over which the diffusion occurs.

To demonstrate the impact of these parameters, pattern dimensions (full-width at half-maximum (FWHM) of $\beta$-phase PL profiles) and contrast (maximum induced $\beta$-phase fraction) were extracted as function of $v$ and $P$ (PL profiles are shown in Supplementary Fig. $2 \mathrm{~b}, \mathrm{c})$. The $\beta$-phase fraction, $\beta$, was estimated by mapping the Raman intensity ratio, $r_{\mathrm{R}}$, of the $1257 \mathrm{~cm}^{-1}$ and $1606 \mathrm{~cm}^{-1}$ modes across the line patterns and using the empirical relation $\left(r_{\mathrm{R}}=3.4 \times 10^{-3} \beta+6.0 \times 10^{-2}\right)$ reported between the two variables ${ }^{9}$. An exemplary $r_{\mathrm{R}}$ map is shown in Fig. $2 b$, with representative Raman spectra given in Supplementary Fig. 2d. The results are presented in Fig. 2c, $d$ and highlight the correlated effects of $P$ and $v$ in the simultaneous tuning of pattern 
a

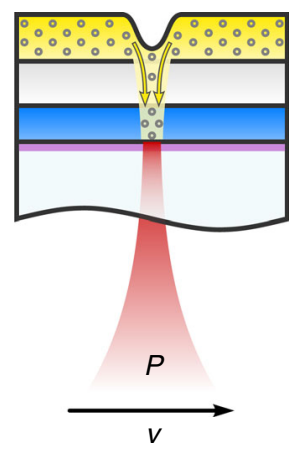

b

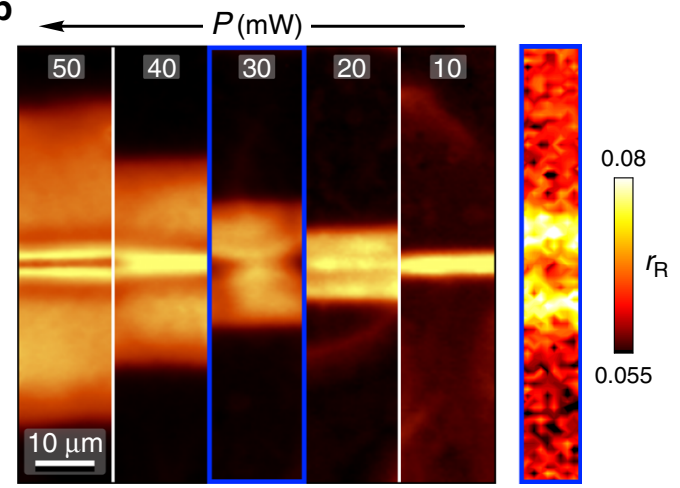

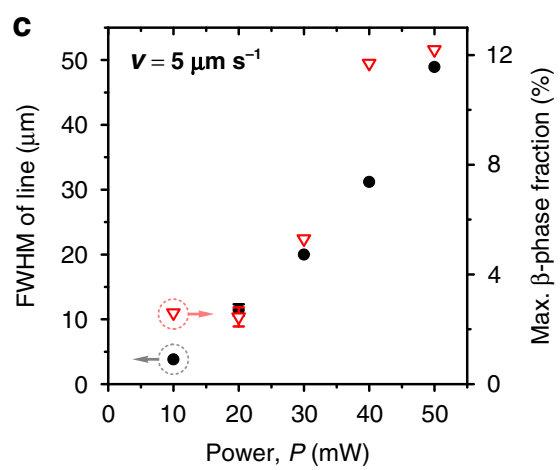

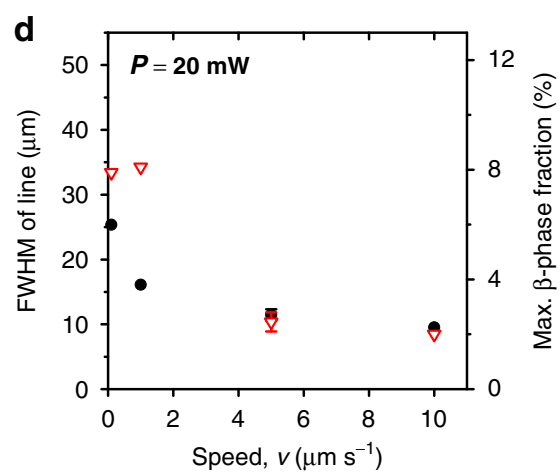

Fig. 2 Chain-conformation patterning in PFO films. a Schematic illustration of the laser-patterning process. Colours and symbols as in Fig. 1. ITO layer is shown by a purple stripe; yellow arrows indicate diffusion of donor. b Confocal PL microscopy images of $\beta$-phase lines patterned in glassy PFO using laser excitation at $785 \mathrm{~nm}$, constant writing speed $v=5 \mu \mathrm{m} \mathrm{s}^{-1}$ and laser power $P=10-50 \mathrm{~mW}$ (as indicated). PL intensity is shown at $438 \mathrm{~nm}$ to spectrally select $\beta$-phase emission. Also shown in $\mathbf{b}$ is an exemplary Raman intensity ratio image (right panel) of the line patterned using $P=30 \mathrm{~mW}$, recorded as the ratio of Raman intensities, $r_{R}$, at 1257 and $1606 \mathrm{~cm}^{-1}$, from which local $\beta$-phase fraction is estimated. c, d FWHM of $\beta$-phase line patterns (black dot, left ordinates) and maximum induced $\beta$-phase fraction in the patterns (red triangle, right ordinates) as function of (c) laser power for $v=5 \mu \mathrm{m} \mathrm{s}{ }^{-1}$ and (d) writing speed for $P=20 \mathrm{~mW}$.

dimensions and contrast. For instance, reducing laser power from 50 to $10 \mathrm{~mW}$ for a constant $v=5 \mu \mathrm{m} \mathrm{s}^{-1}$ (as in Fig. 2b) enables a ten- and sixfold reduction of, respectively, the width and contrast of $\beta$-phase patterns.

Due to the increase of refractive index resulting from the glassy-to- $\beta$-phase conformational transition ${ }^{8}$, the demonstrated $\beta$-phase patterning provides a straightforward approach towards the fabrication of photonic structures such as waveguides and refractive index gratings 8,33 , while the use of ITO/glass substrates enables natural integration into optoelectronic devices. A pattern resolution of $\sim 4 \mu \mathrm{m}$ is achieved despite limiting factors such as lateral heat propagation within ITO and dispersive diffusion of LA within the relatively thick $(\sim 200 \mathrm{~nm})$ gate interlayer. This resolution is $\sim 50$ times better than was obtained previously with vapour printing ${ }^{33}$ and only a factor of $\sim 4$ larger than demonstrated for dip-pen nanolithography ${ }^{8}$ - a technique for which patterning speeds are generally limited to $<1 \mu \mathrm{m} \mathrm{s}^{-1}$. Moreover, further improvements can be enabled by addressing the above-mentioned issues, with additional tuning of laser excitation required to prevent overheating-induced 'train-rail' profiles evident for the lines written at high $P$ (Fig. $2 b$ and Supplementary Fig. 2b, c).

Finally, given the polymer: solvent co-crystal structure of $\beta$ phase PFO one can estimate the amount of LA that was diffused into the film during patterning. Using the known molar volume of LA and the 'cavity' volume for $\beta$-phase $\mathrm{PFO}^{34}$, a stoichiometry of $1: 1$ is predicted, implying that for the patterns with the lowest achieved $\beta$-phase fraction of $\sim 2 \%$ the composition of LA molecules to PFO repeat units is $\sim 1: 50$. (In practice, even lower $\beta$-phase fractions can be obtained, although it is noted that quantifying them by spectroscopic Raman mapping becomes increasingly prone to uncertainties.) Considering that full optimisation was not undertaken, such fine control of the diffusion rate clearly exemplifies the aforementioned moleculeon-demand concept.

Patterning chain orientation. Besides enabling intra-molecular rearrangement, as in the previous examples, certain crystallisable solvents can also induce directional orientation of semicrystalline polymers by epitaxial solidification ${ }^{14,15,28}$. The principal requirements are the crystal lattice match between the fast growth axis of the small-molecular compound and the $c$-axis of the polymer, and the crystallisation of the small-molecular compound preceding that of the polymer ${ }^{28}$. The first of these can be satisfied by selection of appropriate small molecules, whereas the second is accomplished by employing, for instance, hypoeutectic compositions.

Here we demonstrate laser patterning of directional chain orientation in isotropic films of poly(3-hexylthiophene) (P3HT) - one of the most widely studied semiconducting polymersusing the above-described approach combined with moleculargate-based processing. 2,1,3-benzothiadiazole (BT) is selected as the crystallisable solvent instead of the more commonly used 1,3,5-trichlorobenzene $e^{14,15,28}$, due to its comparable periodicity along the fast growth axis $\left(c_{\mathrm{BT}}=3.85 \AA ; c_{\mathrm{P} 3 \mathrm{HT}} / 2 \approx 3.8 \AA\right)^{14,37}$ and a lower melting temperature $\left(T_{\mathrm{m}} \approx 44^{\circ} \mathrm{C}\right)$, which facilitates patterning by laser-induced heating. Additional information on 

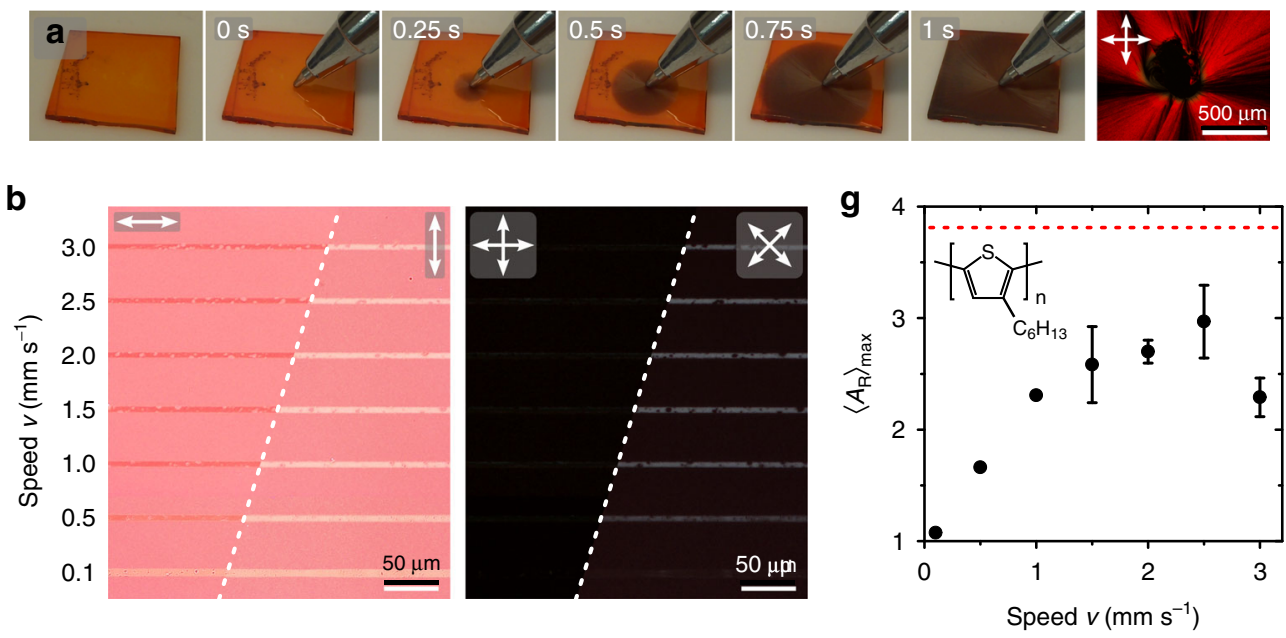

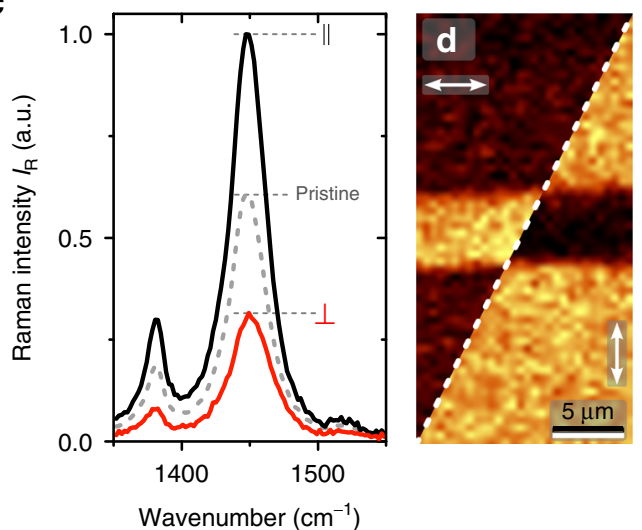

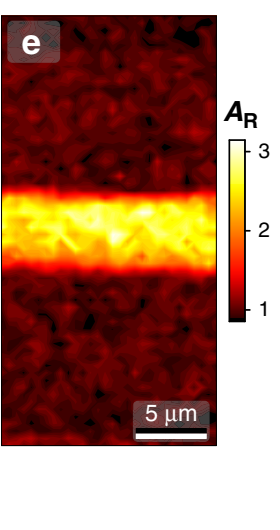

Fig. 3 Chain orientation patterning in P3HT films. a Directional crystallisation of a P3HT : BT film by nucleation with BT. Photographs are shown at the indicated times. Also shown is a cross-polarised micrograph recorded at the centre of the obtained P3HT film following sublimation of BT. $\mathbf{b}$ Transmittedlight micrographs of a P3HT film laser-patterned at the indicated writing speeds $v$. Polarised-incidence and cross-polarised micrographs are shown, with the corresponding relative orientations of polariser transmission axes indicated by the arrows. c-f Exemplary spatially resolved Raman spectroscopy analysis of the line patterned using $v=2.5 \mathrm{~mm} \mathrm{~s}^{-1}$. c Polarised Raman spectra centred on the symmetric $\mathrm{C}=\mathrm{C}$ stretching mode of the $\mathrm{P} 3 \mathrm{HT}$ recorded at the centre of the pattern (black and red lines) and pristine unpatterned area (dotted grey line). $\mathbf{d}$ Polarised Raman maps showing maximum intensity $I_{R}$ at $\sim 1450 \mathrm{~cm}^{-1}$ recorded for the indicated excitation/detection polarisations, and (e) the corresponding Raman anisotropy image $\left(A_{R}=I_{R, I} / I_{R, \perp}\right)$. f Average Raman anisotropy $\left\langle A_{R}\right\rangle$ profile across the pattered line. $\mathbf{g}$ Maximum values of $\left\langle A_{R}\right\rangle$ as a function of speed $v$. Dotted red line indicates the corresponding value obtained for a macroscopically oriented film, as in $\mathbf{a}$. Inset shows the chemical structure of P3HT.

composition-dependent dissolution and re-crystallisation of P3HT : BT blends is given in Supplementary Fig. 3.

A proof-of-principle demonstration of BT-induced orientation of P3HT is shown in Fig. 3a for a freshly spin-coated P3HT : BT blend film (see Supplementary Movie 2). Initially, the film comprises a thin layer of a supercooled solution displaying the characteristic orange colour of dissolved P3HT. Creating a nucleation site-here simply by touching the film with a ballpoint pen at time $t=0 \mathrm{~s}$-initiates radial crystal growth of BT and, therewith, P3HT at a speed of $\sim 8 \mathrm{~mm} \mathrm{~s}^{-1}$ (note the emergence of dark-red colour of crystalline P3HT). The crosspolarised micrograph recorded at the centre of the film following removal of BT by sublimation confirms radial chain orientation of P3HT (Fig. 3a).

To demonstrate fine spatial control of chain orientation, we again utilise the molecular-gate concept. In the following, the sample geometry for laser patterning using excitation at $785 \mathrm{~nm}$ consists of a P3HT film on ITO/glass substrate, capped with a pNaSS film and overlaid on top with a solid BT layer (full details in the 'Methods' section).
Polarised transmitted-light micrographs of patterned P3HT films (Fig. 3b) clearly indicate optical anisotropy within the line patterns obtained at $v \geq 0.5 \mathrm{~mm} \mathrm{~s}^{-1}$, for which higher absorbance parallel to the long axis indicates preferential orientation of chains, and the corresponding average dipole moments, along the laser scanning direction. Cross-polarised micrographs (Fig. 3b) confirm the presence of birefringence arising from directional crystallisation of P3HT. Additional large-area micrographs highlighting the homogeneity of patterned features are given in Supplementary Fig. 4.

Detailed analysis of molecular anisotropy of P3HT within the patterns is performed using polarised spectroscopic Raman mapping of the $1450 \mathrm{~cm}^{-1}$ mode $(\mathrm{C}=\mathrm{C}$ in-plane symmetric stretching) characteristics. Figure $3 \mathrm{c}$ shows exemplary Raman spectra recorded at the centre of a line pattern, revealing higher Raman intensity for polarisation parallel to the writing directionconsistent with the results of optical microscopy. A $\sim 2 \mathrm{~cm}^{-1}$ shift of the $1450 \mathrm{~cm}^{-1}$ mode to higher energies for the perpendicularpolarised Raman spectrum corroborates the presence of predominantly disordered chain segments ${ }^{15}$ perpendicular to the 
writing direction. Raman anisotropy, $A_{\mathrm{R}}$, was quantified as the ratio of maximum intensities, $I_{\mathrm{R}}$, at $\sim 1450 \mathrm{~cm}^{-1}$ recorded with polarisations parallel/perpendicular to the writing direction. Figure $3 \mathrm{~d}-\mathrm{f}$ illustrate the analysis steps, namely the $I_{\mathrm{R}}$ maps for the two different polarisations (Fig. $3 \mathrm{~d}$ ) and the resulting $A_{\mathrm{R}}$ map (Fig. 3e), from which the profile of average $A_{\mathrm{R}}$ across the pattern is calculated (Fig. 3f).

The maximum value of average Raman anisotropy, $\left\langle A_{R}\right\rangle_{\max }$, for the patterns is plotted as a function of $v$ in Fig. $3 \mathrm{~g}$, providing further insight into the patterning process. $\left\langle A_{\mathrm{R}}\right\rangle_{\max }$ peaks at $\sim 3$ for $v=2.5 \mathrm{~mm} \mathrm{~s}^{-1}$, with higher speeds leading to a roll-off due to an insufficient temperature rise and/or limited diffusion of BT across the gate, whereas lower speeds result in depletion of BT from the illuminated area leading to isotropic crystallisation of P3HT. The highest value of $\left\langle A_{\mathrm{R}}\right\rangle_{\max }$ obtained within the patterns (Fig. 3g) is somewhat lower than that recorded for a macroscopically oriented film, indicating the possibility of further improvements.

Hence, laser patterning of chain orientation is demonstrated for a model semicrystalline semiconducting polymer, yielding features with $\mathrm{FWHM} \approx 4.6 \pm 0.3 \mu \mathrm{m}$ written at speeds up to $3 \mathrm{~mm} \mathrm{~s}^{-1}$. Such structures present opportunities for local, micrometre-scale engineering of optical, electronic and thermaltransport properties in thin-film optoelectronic devices, as well as further applications such as security features, of which Fig. 3b presents a first example. We note that the use of the molecular gate is the key enabling feature in the demonstrated process, with its absence-or insufficient thickness-resulting in random patterns of orientation dictated by that of the crystalline BT donor layer (Supplementary Fig. 5). Topography analysis of line patterns of chain orientation by atomic force microscopy (AFM) reveals only a minor $\sim 2 \%$ reduction of the total film thickness in the patterned regions resulting from the densification that accompanies polymer crystallisation (Supplementary Fig. 6). This stands in stark contrast to RMS roughness values $\sim 30 \mathrm{~nm}^{14}$, and in some cases exceeding $100 \mathrm{~nm}^{15}$, for P3HT films conventionally oriented using solid solvents, highlighting the confinement action $^{38}$ provided by the molecular gate, which would facilitate integration of such patterned films into devices.

Patterning local doping. Although in the previous examples the functional small molecules were removed after patterning by spin-off or sublimation, here we seek to pattern material composition by retaining the small-molecular component. As a relevant and timely example ${ }^{39}$, we use patterning of electrical conductivity in the high-mobility semiconductor poly(2,5bis(3-tetradecylthiophen-2-yl)thieno-[3,2-b]thiophene) (PBTTT) by $p$-doping with the Lewis acid tris(pentafluorophenyl)borane $(\mathrm{BCF})^{40}$ (chemical structures in Fig. $4 \mathrm{a}$ ). BCF has been previously reported to be an effective dopant for $\mathrm{P}_{3} \mathrm{HT}^{41}$ and other (macro-) molecular semiconductors ${ }^{42}$, but, to our knowledge, has not been used with PBTTT to date.

As in the previous examples, heating a trilayer structure comprising PBTTT, molecular-gate and the small-molecular BCF dopant induces diffusion of BCF across the gate and doping of the semiconducting polymer layer. The conductivity of PBTTT as a function of annealing temperature $T$ follows a sigmoidal evolution (Fig. 4b; measured following spin-off of the auxiliary layers) and reaches a maximum value of $62 \mathrm{~S} \mathrm{~cm}^{-1}$, surpassing $\sim 4 \mathrm{~S} \mathrm{~cm}^{-1}$ obtained for the similarly straightforward solution-based doping using the more common molecular acceptor 2,3,5,6-tetrafluoro7,7,8,8-tetracyanoquinodimethane $\left(\mathrm{F}_{4} \mathrm{TCNQ}\right)^{43,44}$. (Note that higher conductivities can be achieved for PBTTT: $\mathrm{F}_{4}$ TCNQ at a macroscopic level via manipulation of blend microstructure, doping mechanism ${ }^{45}$ and molecular orientation ${ }^{46}$, as well as the use of vapour-phase doping ${ }^{44,45}$.) Two further features of these results are highlighted for their role in enabling the patterning presented below. First, in the absence of annealing, the PBTTT film retains its low conductivity (Fig. 4b). Second, the spin-off procedure does not appreciably de-dope PBTTT, as illustrated in Supplementary Fig. 7.

The corresponding transmitted-light micrographs (Fig. 4c) evidence a progressive colour change from red to faint-pink upon doping, providing a visual indication of increased electrical conductivity of PBTTT. This change can be understood by reference to the corresponding absorption spectra (Supplementary Fig. 8) showing the emergence of a broad feature centred at $\sim 830 \mathrm{~nm}$ (PBTTT cations and BCF anions) ${ }^{41}$ and simultaneous attenuation of the peak at $\sim 552 \mathrm{~nm}\left(S_{0}-S_{1}\right.$ absorption of PBTTT), with an isosbestic point located at $637 \mathrm{~nm}$.

To estimate the conductivity within the patterns (vide infra), an indirect analysis using spectroscopic Raman mapping was employed. Raman spectra of reference macroscopically doped PBTTT films (as in Fig. 4b, c) were recorded over large, $\sim 4 \times 4$ $\mathrm{mm}^{2}$ sample areas, to minimise uncertainties arising from any inhomogeneities at intermediate $T$ (cf. Fig. 4c) and to provide a better correlation with the large-area conductivity measurements. Selected Raman spectra as a function of conductivity are shown in Fig. 4d. Previous reports have noted the doping-induced increase in the Raman intensity ratio, $r_{\mathrm{R}}$, of the $1393 \mathrm{~cm}^{-1}$ mode (thiophene $\mathrm{C}-\mathrm{C}$ stretch) relative to the $1417 \mathrm{~cm}^{-1}$ mode (thienothiophene $\mathrm{C}=\mathrm{C}$ stretch) ${ }^{47,48}$. Hence, we use $r_{\mathrm{R}}$ plotted against conductivity $\sigma$ (Fig. $4 \mathrm{e}$ ) as a reference for subsequent analysis, whereby a fit of the data using an empirical equation $r_{\mathrm{R}}=A \sigma^{b}+C \quad(A=0.23, \quad b=0.39$ and $C=0.84)$ provides a 'calibration' for $\sigma$ from $r_{\mathrm{R}}$.

Unlike in the previous examples, here patterning via laserinduced heating is enabled by resonant excitation at $532 \mathrm{~nm}-$ close to the absorption peak of PBTTT (Supplementary Fig. 8). Figure $4 \mathrm{f}$ shows that the conductivity obtained within patterned regions as function of laser power $P$ and speed $v$ exhibits the expected dependency, with higher $P$ yielding increased conductivity at highest $v$ due to enhanced temperature rise within the exposure time. The saturation and eventual roll-off for low $v$, on the other hand, are attributed to excessive temperature rise and, therewith, de-doping of PBTTT (see Supplementary Fig. 9 for illustration of the above). A closer look at pattern dimensions as function of $P$ and $v$ provides further insight into the patterning process. As shown in Supplementary Fig. 10b and Supplementary Table 1, reducing writing speed (i.e., increasing the effective diffusion time) by a factor of 600 leads only to a minor factor of 1.6 average increase in pattern dimensions. This indicates that the parasitic in-plane diffusion component is relatively small, as can be expected from an Arrhenius-type exponential dependence of diffusion coefficient on temperature ${ }^{36}$, with the latter decreasing sharply outside the laser-illuminated area. On the contrary, increasing laser power by a factor of 2 leads to a factor of 2.2 average increase in pattern dimensions, indicating that the laserheating-induced temperature rise is the primary parameter governing pattern dimensions.

As a relevant example of potential applications, Fig. $4 \mathrm{~g}$ shows PBTTT patterned with a radio-frequency identification (RFID)antenna-type structure, with additional analysis given in Fig. $4 \mathrm{~h}$, i. FWHM dimensions of the individual lines are $18 \mu \mathrm{m}$, with maximum conductivity reaching $\sim 3 \mathrm{~S} \mathrm{~cm}^{-1}$, whereas the film areas outside the pattern retain the low conductivity of pristine PBTTT (Fig. 4i).

Here we note yet another important enabling feature of the molecular-gate concept, namely that the presence of the hydrophilic pNaSS interlayer, in fact, provides optimal wetting and facilitates deposition of homogeneous BCF donor layers from 
a

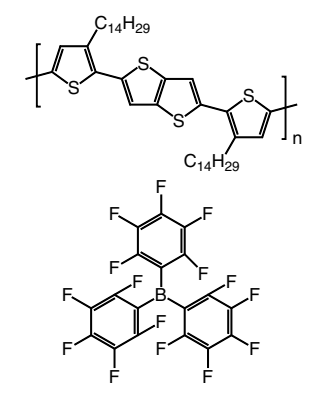

b

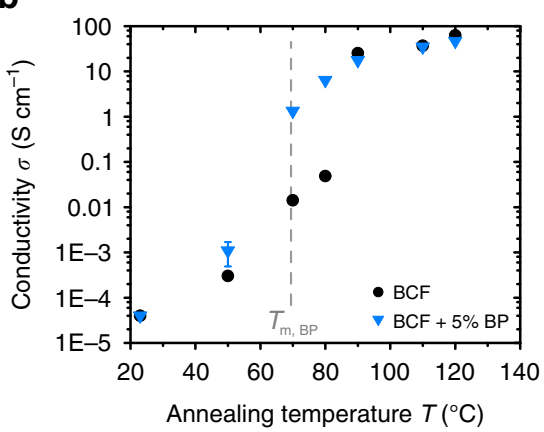

C

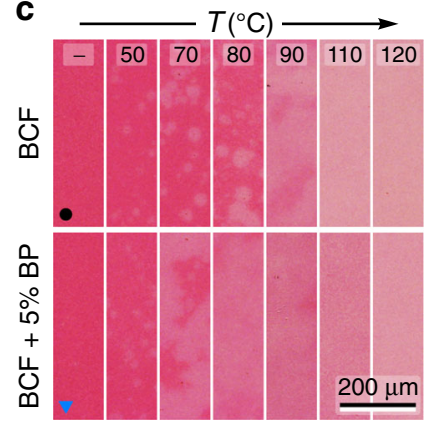

d

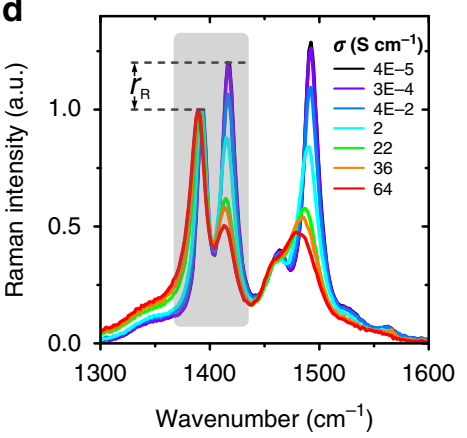

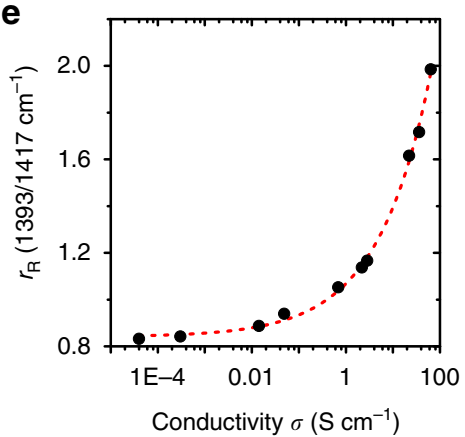

f

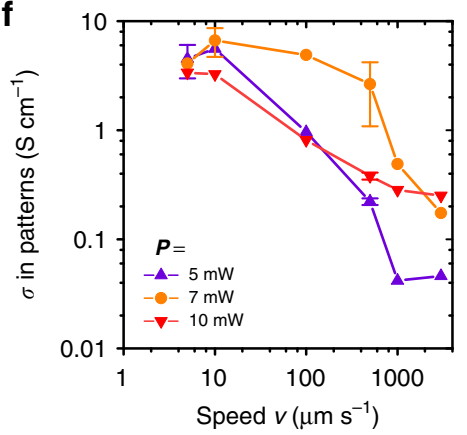

g

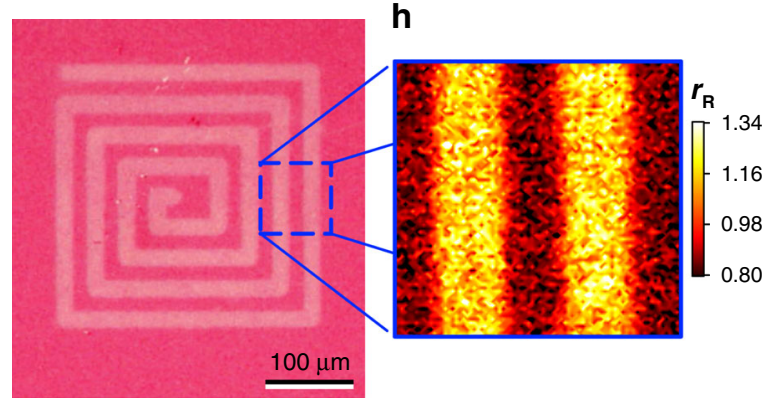

i

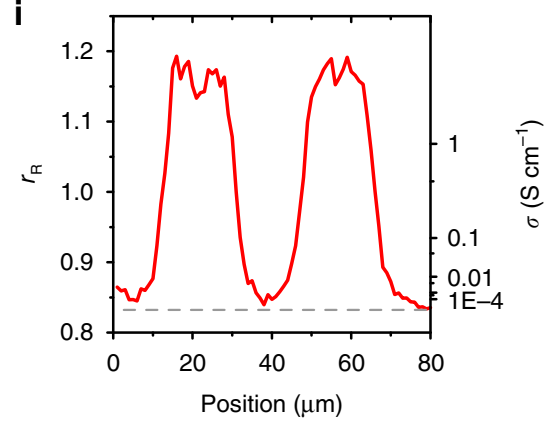

Fig. 4 Electrical conductivity patterning in PBTTT films by local doping. a Chemical structures of PBTTT and BCF. $\mathbf{b}$ Conductivity $\sigma$ of PBTTT films doped through gate by annealing for $1 \mathrm{~min}$ at temperature $T$. Solution-deposited dopant layers comprised BCF (black dot) and BCF +5 wt $\%$ biphenyl ('BP'; blue triangle) added as a co-solvent. Dashed line indicates the melting temperature of BP. c Transmitted-light micrographs for the films in $\mathbf{b}$. $\mathbf{d}$ Selected Raman spectra of doped PBTTT films, with conductivities indicated. Spectra are normalised by the intensity of the peak at $\sim 1393 \mathrm{~cm}^{-1}$; shaded region highlights the peaks used for estimating conductivity. e Raman intensity ratio $r_{R}$ of the maximum intensities of the $\sim 1393 \mathrm{~cm}^{-1}$ and $\sim 1417 \mathrm{~cm}^{-1}$ peaks of PBTTT as a function of $\sigma$. Dotted line indicates a curve fit to the data. $\mathbf{f}$ Maximum conductivities obtained in line patterns of doping induced by scanning laser excitation at $532 \mathrm{~nm}$ for the indicated incident power $P$ and writing speed $v$. Conductivities were extracted by Raman mapping of $r_{\mathrm{R}}$ using the calibration data in $\mathbf{e}$. $\mathbf{g}$ Transmitted-light micrograph of laser-patterned $\left(P=7 \mathrm{~mW}, v=3 \mu \mathrm{m} \mathrm{s}^{-1}\right)$ RFID-antenna-type structure. $\mathbf{h}$ Raman map of $r_{\mathrm{R}}$ for the indicated area and (i) the corresponding average profiles of $r_{\mathrm{R}}$ (left ordinate) and $\sigma$ (right ordinate).

solutions in polar solvents. On the other hand, conventional deposition directly onto the hydrophobic PBTTT surface yields strongly dewetted BCF overlayers that are of little practical use (Supplementary Fig. 11).

Overall, the demonstrated molecular-gate-based approach to patterning electrical conductivity (and, more generally, material composition) opens numerous avenues for applications, being simpler than the established methods which typically rely on nontrivial photochemistry or require post-patterning back-filling with pristine material ${ }^{4,49-51}$. PBTTT films doped via molecular gate exhibit excellent long-term stability, with their electrical conductivity remaining essentially unchanged after aging for six months (Supplementary Fig. 12). More generally, in the absence of specific host-donor interactions as in the present example, the thermal stability of material composition patterns would be governed by the glass transition temperature $\left(T_{\mathrm{g}}\right)$ of the polymer ${ }^{52}$ and the resulting $T_{\mathrm{g}}$ of the polymer-donor blend ${ }^{53}$, which can be optimised for specific applications via material selection.

Further optimisation of the process is possible, as evidenced by the highest laser-patterned conductivities falling short of those recorded for macroscopically doped samples (cf. Fig. 4b, f). A promising approach may involve the addition of a small amount of a solid 'co-solvent' to BCF ( $+5 \mathrm{wt} \%$ biphenyl (BP)), which, as shown in Fig. 4b, $c$ and Supplementary Fig. 13, lowers the annealing temperature and time required for reaching a given conductivity by enhancing diffusion of BCF through the gate.

Beyond 'binary' patterning. The above-demonstrated use of multiple small-molecular compounds (i.e., BCF + BP) comprising the donor layer can be extended to provide on-demand patterning of several types of functionalities in a single processing 

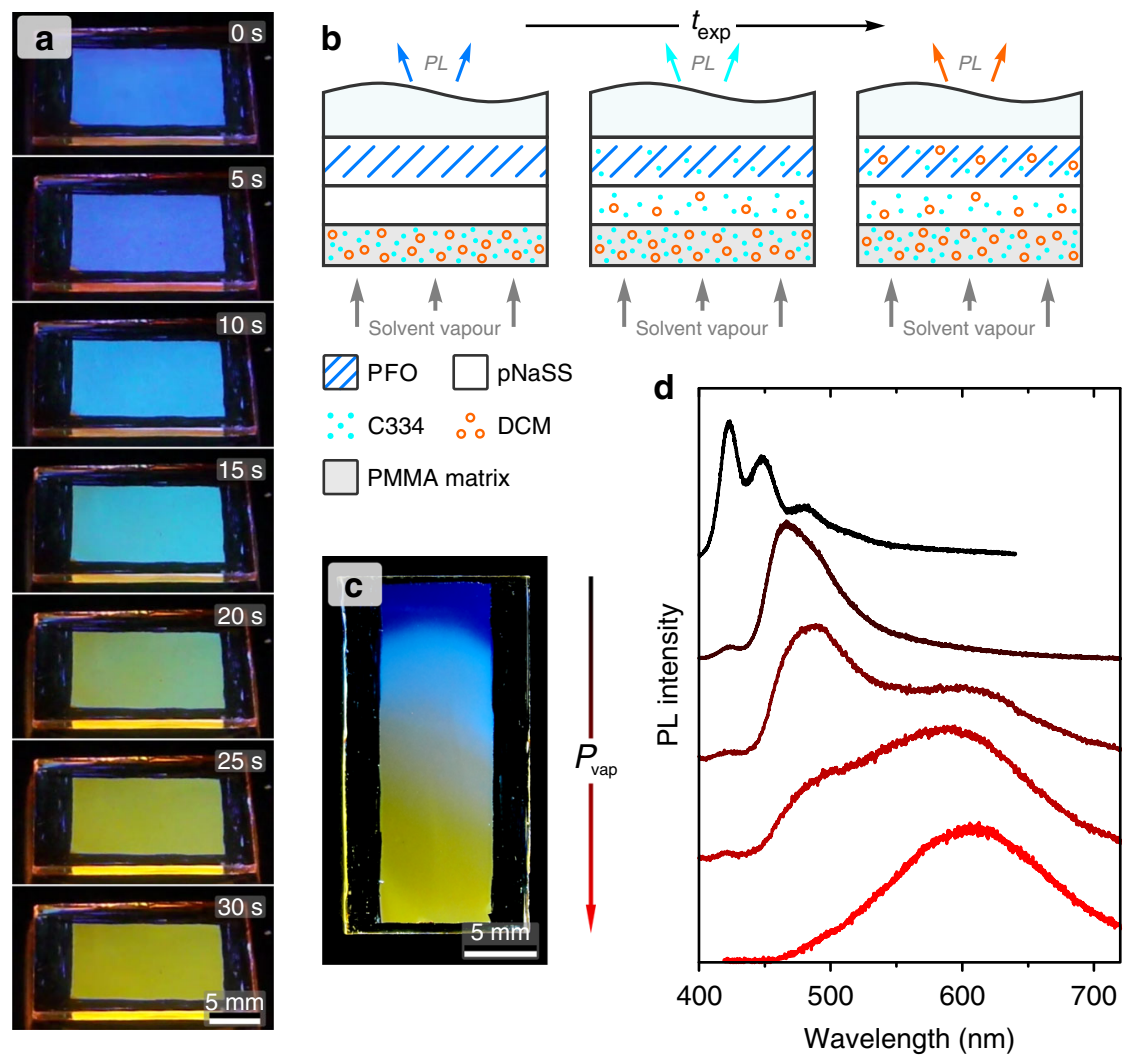

Fig. 5 One-step broad PL emission tuning in PFO-based blend films. a Photographs of an inverted PFO/pNaSS/dye-blend trilayer on a glass substrate taken under UV illumination during exposure to 1:1 acetone: water vapour at the indicated times. $\mathbf{b}$ Schematic illustration of the process in $\mathbf{a}$, showing the solvent-vapour-induced (quasi-)sequential diffusion of the cyan- and orange-emitting dyes with increasing exposure time, $t_{\exp }$. $\mathbf{c}$ Photograph of a similarly processed PFO-based blend film but with relative solvent vapour pressure, $P_{\text {vap }}$, increasing from top to bottom of the sample, resulting in a gradient of $\mathrm{PL}$ emission colours. d PL spectra recorded for the sample in c spanning positions from top to bottom.

step-i.e., without the need for time-consuming post-processing or re-alignment. This approach relies on identifying smallmolecular compounds with desired functionalities, and said compounds possessing sufficiently different physico-chemical properties to enable their (quasi-)discrete, time-separated diffusion through the gate.

Here we demonstrate this concept by broad tuning of PL emission from an active layer via control of its ternary composition. It is well known that adding small amounts of a green-emitting dye to a blue emitter with cascading energy levels can lead to energy transfer from the blue to the green emitter and therewith greendominated emission. This can be extended to red emission with an appropriate choice of dyes ${ }^{54}$. As an example, we show broad PL tuning in PFO-based blend films. Here the donor layer deposited onto the molecular gate comprises a $2: 1 \mathrm{wt} / \mathrm{wt}$ blend of Coumarin 334 ('C334'; cyan-emitting dye) and 4-(dicyanomethylene)-2methyl-6-(4-dimethylaminostyryl)-4H-pyran (DCM) (orange-emitting dye) (chemical structures and reference PL spectra are shown in Supplementary Fig. 14). The donor layer additionally contains mandelic acid (solid co-solvent) and poly(methyl methacrylate) (PMMA), with the latter added to prevent dye-blend dewetting (Supplementary Fig. 15). Diffusion of the dyes into PFO is then enabled by exposure of the trilayer structure to a vapour of acetone: water (as in Fig. 1b). Of the selected dyes, C334 has a somewhat smaller molar volume than DCM as inferred from their molar masses (283.3 and $303.4 \mathrm{~g} \mathrm{~mol}^{-1}$ respectively), as well as higher relative solubility in acetone due to the presence of carbonyl groups in its chemical structure. Hence, C334 can be expected to diffuse substantially faster than DCM via the gate into the PFO layer, satisfying the requirements for (quasi-)discrete patterning of the two dyes.

Images of the trilayer structure under UV light illumination taken in real time during continuous exposure to solvent vapour are shown in Fig. 5a, with the process illustrated schematically in Fig. $5 \mathrm{~b}$ (see also Supplementary Movie 3). Initially (time $t=0-5$ s), the sample exhibits the characteristic blue PL of PFO. It is noteworthy that at this stage the PL of dye-blend layer is not apparent due to the fact that the images are recorded for the inverted sample (with PFO facing up), as well as concentrationinduced quenching of dye PL. Subsequently the PL of the sample turns cyan $(t=10-15 \mathrm{~s})$ due to diffusion of C334 in PFO and the resulting $\mathrm{PFO} \rightarrow \mathrm{C} 334$ energy transfer occurring for excitation at $365 \mathrm{~nm}$. Finally $(t=25-30 \mathrm{~s})$, the PL of the sample turns orange, signifying diffusion of DCM into the PFO layer, with the emission governed by $\mathrm{PFO} \rightarrow(\mathrm{C} 334 \rightarrow) \mathrm{DCM}$ energy transfer.

As a patterning example for the approach presented above, Fig. 5c shows an image of a PFO-based blend film (following removal of the dye-blend donor layer) under UV light illumination that was patterned by solvent vapour exposure through a slot-die placed at the lower end of the substrate. In this configuration, the relative solvent vapour pressure $P_{\text {vap }}$ (i.e., ratio of vapour pressure at a given location on the sample to that of the saturated stream) varies across the sample, producing a gradient of PL emission colour. Locally acquired PL spectra (Fig. 5d) exhibit position-dependent variation of the relative amplitudes of individual spectral contributions, with progressive quenching of PFO and C334 emission with increasing $P_{\text {vap }}$ due to excitation energy transfer to the energetically lower-lying species. 


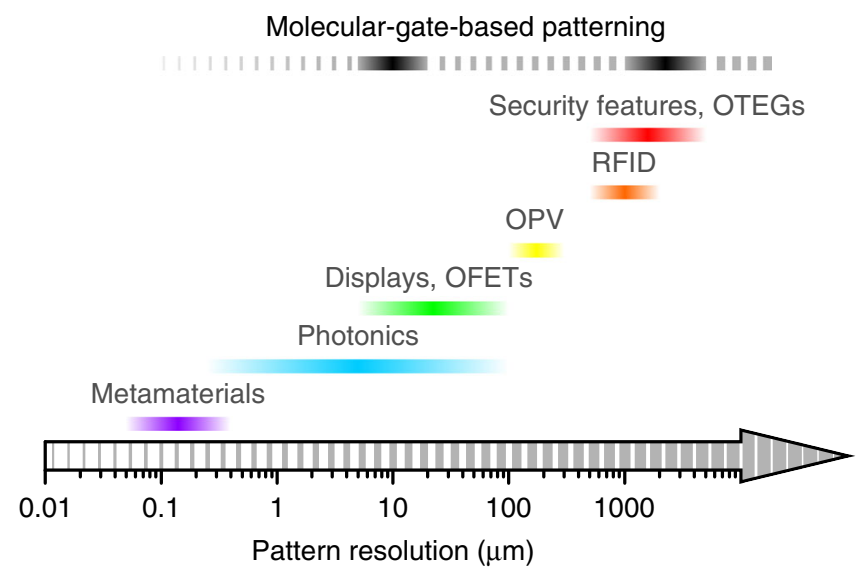

Fig. 6 Typical length scales required for patterning organic

semiconductors. The characteristic dimensions are shown for metamaterials, photonic structures, displays, organic field-effect transistors (OFETs), organic photovoltaics (OPV), RFID tags, security features and organic thermoelectric generators (OTEGs). Pattern resolutions obtained in the present work are indicated by solid black bars, with broken bars showing their plausible extensions.

Comparison with state-of-the-art methods. Conceptually, the closest analogue to the described method in the state-of-the-art is LIFT $^{17}$ and its variants such as laser-induced thermal imaging $(\text { LITI })^{18,55}$ in which optical heating induces material transfer between a donor sheet and a target substrate placed in close proximity or direct contact. The distinguishing features of molecular-gate-based patterning with respect to LIFT and LITI include lower laser powers, avoiding material degradation inherent to laser-induced donor vaporisation in LITI, moleculeby-molecule rather than dropwise material transfer that offers finer process tuneability, and the capability of patterning both microstructure and material composition. A further difference with most state-of-the-art techniques-which involve physical (additive or subtractive) patterning to produce bilayer-type structures-is that the demonstrated patterning method retains film planarity by donor incorporation within a target film. While retaining the planar film format is advantageous for straightforward integration into multilayer structures, its drawbacks may include issues such as cross-talk which, for instance in the case of OLED displays, requires physical separation between adjacent pixels. One may envisage, however, that leakage can be minimised when the method is combined with laser ablation or for particular geometries given substantial contrast between patterned and baseline regions in terms of, e.g., electrical conductivity (doping patterns) or charge-carrier mobility (chain orientation patterns).

In terms of throughput, for instance in the context of OLEDs, typical material deposition speeds by inkjet printing, slot-die coating and gravure printing are in the $0.1-5 \mathrm{~m} \mathrm{~s}^{-1}$ range $^{23}$. Evidently, the speeds used in this first demonstration of molecular-gate-based patterning $\left(\leq 3 \mathrm{~mm} \mathrm{~s}^{-1}\right)$ fall short of those for these established techniques. Further optimisation of laser excitation by using high-power sources split into multiple, individually addressable spots as employed in LITI $^{18}$ may bring the throughput of the method to the moderate values of $\sim 0.1 \mathrm{~m}^{2}$ $\min ^{-1}$ associated with laser-based thermal transfer methods ${ }^{6}$, while even higher throughputs are common in other state-of-theart laser structuring methods ${ }^{56,57}$. By comparison, typical throughput values for inkjet printing ${ }^{2}$ are $\sim 30 \mathrm{~m}^{2} \mathrm{~min}^{-1}$. Nevertheless, the use of solution-based deposition and removal of auxiliary layers implies that molecular-gate-based patterning can be configured as a serial process, rather than a batch-type LITI process, and therefore be compatible with roll-to-roll fabrication $^{58}$. The use of co-solvents (as demonstrated for the case of doping; see Fig. 4b and Supplementary Fig. 13) may be further optimised to improve throughput.

Applications. The field of molecular electronics and photonics offers numerous potential applications for the demonstrated method: from the design of photonic structures via chainconformation patterning ${ }^{8,33}$ and displays ${ }^{59,60}$ to the fabrication of high-performance field-effect transistors and organic thermoelectric generators by harnessing the capability for local doping $^{61,62}$, as well as manipulating electronic ${ }^{11,63}$ and thermal ${ }^{13}$ conductivities via molecular orientation. Figure 6 shows typical feature sizes required for a selection of electronic and photonic devices and structures. With patterning on $4-25 \mu \mathrm{m}$ and millimetre scales demonstrated herein, the molecular-gate-based approach appears to cover most of the range. Extensions to intermediate and larger scales can be plausibly enabled by modification of stimulus to de-focussed laser beams, solvent vapour jets and heated stamps. Employing deep UV excitation, as in state-of-the-art photolithography, as well as using immersion objectives can enable resolutions $<1 \mu \mathrm{m}$. Stimuli such as electron beams and heated AFM tips may enable feature sizes down to the thickness of the molecular gate itself $(\sim 100 \mathrm{~nm})$, albeit with inevitably compromised throughput.

Given the generality of the underlying principles, applications can extend to other fields. The small-molecular donor layer may comprise compounds such as catalysts, cross-linkers or, in fact, reactive monomers for subsequent polymerisation into functional superstructures ${ }^{64}$ within the target film. Similarly, the target film can take form of a wide range of (macro-)molecular materials. Hence, one may conceive applications such as patterning polarised security features ${ }^{29,65}$ for banknotes and personal ID documents or patterning of antigens in immobilised layers of antibodies for biosensing ${ }^{66}$. Elsewhere, ambient-responsive dyes patterned in commodity polymers can find applications in smart packaging ${ }^{67}$.

\section{Discussion}

In summary, we have demonstrated a versatile method for spatial patterning of microstructure and material composition in organic semiconductors by employing a solution-processed moleculargate interlayer. Patterning of all principal feature types was demonstrated, including chain conformation and orientation, and material composition. Heat-, solvent-vapour- and laser-based implementations were presented, with minimum resolution $<5 \mu \mathrm{m}$ and writing speeds up to $3 \mathrm{~mm} \mathrm{~s}^{-1}$. A number of further advantages of the molecular-gate-based approach were highlighted, such as the capacity for one-step (quasi-)discrete patterning of multiple functionalities or components. The demonstrated method is expected to provide a practical means to micro-patterning of organic semiconductors in both roll-to-roll and laboratory-scale environments for applications in the field of molecular electronics and beyond.

\section{Methods}

Materials. pNaSS (MW $\sim 1 \times 10^{6} \mathrm{~g} \mathrm{~mol}^{-1}$ ), PFO, P3HT (regioregular), PMMA $\left(\mathrm{MW} \sim 15,000 \mathrm{~g} \mathrm{~mol}^{-1}\right)$ and $\mathrm{C} 334$ were purchased from Aldrich. LA $(>98 \%), \mathrm{BT}$ $(>99 \%), \mathrm{BCF}(>98 \%), \mathrm{BP}(>99.5 \%), \mathrm{DCM}$ and $\mathrm{L}-(+)$-mandelic acid $(>99 \%)$ were purchased from TCI Chemicals. PBTTT was purchased from 1-Material. ITO/glass substrates (ITO thickness $\sim 100 \mathrm{~nm}$ ) were purchased from Ossila. All materials were used as received. Details of the employed solvents are given in the Supplementary Note 1 .

Sample fabrication. pNaSS 'molecular-gate' films were spin-coated from 2 to 4 wt $\%$ solutions in deionised water with Triton X-100 surfactant added at $\sim 1 \mathrm{wt} \%$ relative to the amount of solvent. pNaSS solutions were filtered (polyethersulfone (PES), 
$0.45 \mu \mathrm{m}$ pore size) prior to use. For all functional small-molecular donor layers, spincoating of the respective solutions was carried out by dynamic deposition-i.e., onto the substrate at the target spin speed. Laser patterning was carried out using a WITec Alpha 300RA instrument using its stepper-motor-driven stage for scanning the sample in the laser focal plane. Continuous-wave (CW) laser excitation was used throughout.

Patterning chain conformation. PFO films were spin-coated from $2 \mathrm{wt} \%$ solutions in toluene at 2000 r.p.m., with both solution and substrates pre-heated to $70^{\circ} \mathrm{C}$, resulting in $\sim 85 \mathrm{~nm}$-thick glassy films. Glass and ITO/glass substrates were used for macroscopic and local chain-conformation switching, respectively. pNaSS films were spin-coated from $4 \mathrm{wt} \%$ solutions at 3000 r.p.m. (thickness $\sim 200 \mathrm{~nm}$ ). LA donor layers were spin-coated from $20 \mathrm{wt} \%$ solutions in acetone at $\sim 8000$ r.p.m. Diffusion of LA into PFO and, therewith, formation of $\beta$-phase was accomplished macroscopically by heating to $50^{\circ} \mathrm{C}$. Local formation of $\beta$-phase was enabled by exposure to laser excitation $(785 \mathrm{~nm}$; power $P=10-50 \mathrm{~mW})$ focussed through the substrate onto the PFO film and scanned at speeds $v=0.1-10 \mu \mathrm{m} \mathrm{s}^{-1}$ and, elsewhere, by nozzle-based exposure to solvent vapour of a $1: 1 \mathrm{vol} / \mathrm{vol}$ acetone : water solution through which $\mathrm{N}_{2}$ carrier gas was bubbled. Note that, in the absence of LA, neither acetone nor water in liquid or vapour form would induce $\beta$-phase formation, being non-solvents for $\mathrm{PFO}^{34}$. In all cases, LA and pNaSS were removed after patterning by spin-off with, sequentially, acetone, water and-again-acetone.

Patterning chain orientation. Proof-of-principle P3HT : BT blend films were spin-coated from $2.5 \mathrm{wt} \%$ solutions in $2: 1 \mathrm{wt} / \mathrm{wt} \mathrm{BT}$ : ethyl acetate at 2000 r.p.m., with solution and substrates held on hotplates at $120^{\circ} \mathrm{C}$ and $40^{\circ} \mathrm{C}$, respectively, prior to deposition. P3HT films were spin-coated from $2 \mathrm{wt} \%$ solutions in chlorobenzene at 4000 r.p.m., with both solution and ITO/glass substrates pre-heated to $50^{\circ} \mathrm{C}$, yielding $\sim 90 \mathrm{~nm}$-thick films. pNaSS films were spin-coated from $4 \mathrm{wt} \%$ solutions at 7000 r.p.m. (thickness $\sim 130 \mathrm{~nm}$ ). BT donor layers were deposited by casting $\sim 20 \mu \mathrm{L}$ of $40 \mathrm{wt} \%$ solutions in methylene chloride (with $1 \%$ of Triton X100) onto films spinning at $\sim 700$ r.p.m. and, following solvent evaporation over $\sim 60 \mathrm{~s}$, placing a $170 \mu \mathrm{m}$-thick glass coverslip onto the still-liquid BT layer and initiating its crystallisation. The function of the coverslip was to prevent excessively rapid sublimation of BT during subsequent processing. Laser patterning of chain orientation was performed using excitation at $785 \mathrm{~nm}(P=55 \mathrm{~mW})$ focussed through the substrate onto the P3HT film and scanned at $v=0.1-3 \mathrm{~mm} \mathrm{~s}^{-1}$. After patterning, the coverslip was peeled off and, following rapid sublimation of BT under ambient conditions, pNaSS gate was removed by spin-off with water.

Patterning electrical conductivity. PBTTT films were spin-coated from $1.5 \mathrm{wt} \%$ solutions in chlorobenzene at 4000 r.p.m., with both solution and glass substrates pre-heated to $110^{\circ} \mathrm{C}$, resulting in $\sim 43 \mathrm{~nm}$-thick films. The films were subsequently crystallised by annealing at $180^{\circ} \mathrm{C}$ for $30 \mathrm{~min}$ under $\mathrm{N}_{2}$ atmosphere followed by slow cooling to room temperature. pNaSS films were spin-coated from $2 \mathrm{wt} \%$ solutions at $\sim 6000$ r.p.m. (thickness $\sim 80 \mathrm{~nm}$ ). BCF donor layer were spin-coated from $60 \mathrm{wt} \%$ solutions in $10: 1 \mathrm{wt} / \mathrm{wt}$ diethyl ether: methanol at 3000 r.p.m. (doping by thermal annealing) or 9000 r.p.m. (laser-based doping). Elsewhere, $5 \mathrm{wt}$ $\%$ of BP (relative to the amount of solvent) was added to BCF solutions to act as a co-solvent at processing temperatures above its melting temperature $\left(T_{\mathrm{m}}=69^{\circ} \mathrm{C}\right)$. Macroscopic doping was enabled by heating the trilayer samples in air to selected temperatures $\left(50-120^{\circ} \mathrm{C}\right)$ for $1 \mathrm{~min}$. Laser patterning of doping was performed using excitation at $532 \mathrm{~nm}(P=5-12.5 \mathrm{~mW})$ focussed $20 \mu \mathrm{m}$ above the sample surface $(\times 40$ objective; $\mathrm{NA}=0.6)$ to yield larger feature size for improved analysis and visualisation, and scanned at $v$ spanning $5 \mu \mathrm{m} \mathrm{s}^{-1}$ to $3 \mathrm{~mm} \mathrm{~s}^{-1}$. After patterning, BCF and pNaSS were removed by spin-off with, sequentially, acetonitrile and water.

Patterning PL emission in PFO-based blends. PFO films were deposited on glass substrates as described above. pNaSS films were spin-coated from $4 \mathrm{wt} \%$ solutions at 3000 r.p.m. (thickness $\sim 200 \mathrm{~nm}$ ). The dye-blend solution comprised $2 \mathrm{wt} \% \mathrm{C} 334$ (cyan-emitting dye) in $1: 2 \mathrm{wt} / \mathrm{wt}$ acetone : methylene chloride with $1 \mathrm{wt} \% \mathrm{DCM}$ (orange-emitting dye), $1 \mathrm{wt} \%$ mandelic acid (co-solvent; $T_{\mathrm{m}}=135^{\circ} \mathrm{C}$ ) and $4 \mathrm{wt} \%$ PMMA (matrix polymer) added relative to the amount of solvent. The dye-blend solution was spin-coated at 6000 r.p.m. (Quasi)-discrete diffusion of the C334 and DCM dyes into the PFO films was achieved by exposure to vapour of $1: 1$ acetone : water (as above) directed at the sample via a large-opening tube (homogeneous PL modification) or a slot-die (graded PL modification). For the latter example, spinoff of the residual dye-blend donor layer was performed with methylene chloride; pNaSS was not removed to avoid further, spin-off-induced changes in PL due to the non-negligible solubility of C334 in water. Reference C334 and DCM films were spin-coated at 1500 r.p.m. from $0.1 \mathrm{wt} \%$ solutions in $1: 2 \mathrm{wt} / \mathrm{wt}$ acetone : methylene chloride that additionally contained $5 \mathrm{wt} \%$ PMMA (relative to the amount of solvent) to maintain dilution of the dyes in the solid state.

Characterisation. Unless otherwise noted, all characterisation of semiconducting polymer films was performed after removal of any auxiliary layers (i.e., molecular gate and donor layer) by spin-off. Whenever shown, error bars indicate standard deviation (SD) from analysis on different areas of a given sample. WITec Alpha
300RA instrument (reflection geometry, scanning mode) was used for spectroscopic PL $\left(\lambda_{\mathrm{ex}}=355 \mathrm{~nm}\right)$ and Raman mapping analyses (CW excitation in all cases); additional PL spectra were recorded under UV light illumination $\left(\lambda_{\mathrm{ex}} \approx 365\right.$ $\mathrm{nm})$. The excitation wavelengths for Raman analysis were as follows: $488 \mathrm{~nm}$ (PBTTT), $633 \mathrm{~nm}$ (PFO) and $785 \mathrm{~nm}$ (P3HT). Polarised Raman analysis was performed with synchronous adjustment of both excitation and detection polarisation. Incident laser power and integration time were optimised to avoid sample degradation by, e.g., de-doping. Small-area transmitted-light microscopy was performed using a BX51 instrument (Olympus); large-area reflected-/transmittedlight microscopy was performed with a Mantis Elite stereo-microscope (Vison Engineering). Vis-NIR transmission spectra were recorded with a Bruker Vertex 70 FTIR spectrophotometer coupled to a Bruker Hyperion optical microscope. Film thickness was measured using a DektakXT profilometer (Bruker). AFM was performed in tapping mode with a Keysight 5500 instrument (Agilent). Electrical conductivity of doped PBTTT samples was measured with an Ecopia HMS-5000 instrument using the four-probe van der Pauw method for square $\sim 6 \times 6 \mathrm{~mm}^{2}$ film areas with silver paste contacts placed on each corner. For neat, undoped PBTTTthe conductivity of which could not be measured directly-the literature value ${ }^{43}$ of $\sigma=4 \times 10^{-5} \mathrm{~S} \mathrm{~cm}^{-1}$ was assumed. Differential scanning calorimetry (DSC) was performed using a Mettler-Toledo DSC 2 instrument calibrated using indium standards. BT and P3HT were loaded into low-pressure aluminium pans and sealed to prevent evaporation of BT elevated temperatures (confirmed by weighing the pans before and after measurement). Samples were cycled in the -30 to $180^{\circ} \mathrm{C}$ range ( -30 to $260^{\circ} \mathrm{C}$ for neat $\mathrm{P} 3 \mathrm{HT}$ in pierced crucibles under $\mathrm{N}_{2}$ flow) at $10^{\circ} \mathrm{C}$ $\min ^{-1}$.

\section{Data availability}

All data supporting the findings of this study are available within the article and its Supplementary Information, or from the corresponding authors upon reasonable request.

Received: 23 February 2020; Accepted: 24 June 2020; Published online: 17 July 2020

\section{References}

1. Inganäs, O. Organic photovoltaics over three decades. Adv. Mater. 30 , 1800388 (2018).

2. Caironi, M. \& Noh, Y.-Y. (eds.) Large area and flexible electronics (Wiley, 2015).

3. Gaspar, D. J. \& Polikarpov, E. (eds.) OLED Fundamentals: Materials, Devices, and Processing of Organic Light-Emitting Diodes (CRC Press, 2015).

4. Menard, E. et al. Micro- and nanopatterning techniques for organic electronic and optoelectronic systems. Chem. Rev. 107, 1117-1160 (2007).

5. Hiszpanski, A. M. \& Loo, Y.-L. Directing the film structure of organic semiconductors via post-deposition processing for transistor and solar cell applications. Energy Environ. Sci. 7, 592-608 (2014).

6. Kahn, B. E. Patterning processes for flexible electronics. Proc. IEEE 103, 497-517 (2015).

7. Liu, C., Xu, Y. \& Noh, Y.-Y. Contact engineering in organic field-effect transistors. Mater. Today 18, 79-96 (2014).

8. Perevedentsev, A. et al. Dip-pen patterning of poly(9,9-dioctylfluorene) chain-conformation-based nano-photonic elements. Nat. Commun. 6, 5977 (2015).

9. Perevedentsev, A., Chander, N., Kim, J.-S. \& Bradley, D. D. C. Spectroscopic properties of poly(9,9-dioctylfluorene) thin films possessing varied fractions of $\beta$-phase chain segments: enhanced photoluminescence efficiency via conformation structuring. J. Polym. Sci. Part B: Polym. Phys. 54, 1995-2006 (2016).

10. Shi, X. et al. Relating chain conformation to the density of states and charge transport in conjugated polymers: The role of the $\beta$-phase in poly $(9,9-$ dioctylfluorene). Phys. Rev. X 9, 021038 (2019).

11. Sirringhaus, $\mathrm{H}$. et al. Mobility enhancement in conjugated polymer field-effect transistors through chain alignment in a liquid-crystalline phase. Appl. Phys. Lett. 77, 406-408 (2000).

12. Min, S.-Y. et al. Large-scale organic nanowire lithography and electronics. Nat. Commun. 4, 1773 (2013).

13. Patel, S. N. \& Chabinyc, M. L. Anisotropies and the thermoelectric properties of semiconducting polymers. J. Appl. Polym. Sci. 134, 44403 (2017).

14. Müller, C. et al. One-step macroscopic alignment of conjugated polymer systems by epitaxial crystallization during spin-coating. Adv. Funct. Mater. 23, 2368-2377 (2013).

15. Dörling, B. et al. Controlled pinning of conjugated polymer spherulites and its application in detectors. Adv. Opt. Mater. 5, 1700276 (2017).

16. Wallraff, G. M. \& Hinsberg, W. D. Lithographic imaging techniques for the formation of nanoscopic features. Chem. Rev. 99, 1801-1821 (1999). 
17. Serra, P. \& Piqué, A. Laser-induced forward transfer: fundamentals and applications. Adv. Mater. Technol. 4, 1800099 (2019).

18. Blanchet, G. B., Loo, Y. L., Rogers, J. A., Gao, F. \& Fincher, C. R. Large area, high resolution, dry printing of conducting polymers for organic electronics. Appl. Phys. Lett. 82, 463-465 (2003).

19. Street, R. A. et al. Jet printing flexible displays. Mater. Today 9, 32-37 (2006).

20. Shtein, M., Peumans, P., Benziger, J. B. \& Forrest, S. R. Micropatterning of small molecular weight organic semiconductor thin films using organic vapor phase deposition. J. Appl. Phys. 93, 4005-4016 (2003).

21. Noh, Y.-Y., Zhao, N., Caironi, M. \& Sirringhaus, H. Downscaling of selfaligned, all-printed polymer thin-film transistors. Nat. Nanotechnol. 2, 784-789 (2007).

22. Strobel, N., Seiberlich, M., Eckstein, R., Lemmer, U. \& Hernandez-Sosa, G. Organic photodiodes: printing, coating, benchmarks, and applications. Flex. Print. Electron. 4, 043001 (2019).

23. Merklein, L. et al. Comparative study of printed multilayer OLED fabrication through slot die coating, gravure and inkjet printing, and their combination. Colloids Interfaces 3, 32 (2019).

24. Kim, C., Shtein, M. \& Forrest, S. R. Nanolithography based on patterned metal transfer and its application to organic electronic devices. Appl. Phys. Lett. 80, 4051-4053 (2002).

25. Kooy, N., Mohamed, K., Pin, L. T. \& Guan, O. S. A review of roll-to-roll nanoimprint lithography. Nanoscale Res. Lett. 9, 320 (2014).

26. Pierre, A. et al. All-printed flexible organic transistors enabled by surface tension-guided blade coating. Adv. Mater. 26, 5722-5727 (2014).

27. Wolfer, P. et al. Photo-induced molecular alignment of trisazobenzene derivatives. J. Mater. Chem. 21, 4339-4345 (2011).

28. Brinkmann, M., Hartmann, L., Biniek, L., Tremel, K. \& Kayunkid, N. Orienting semi-conducting $\pi$-conjugated polymers. Macromol. Rapid. Commun. 35, 9-26 (2014).

29. Müller, C., Garriga, M. \& Campoy-Quiles, M. Patterned optical anisotropy in woven conjugated polymer systems. Appl. Phys. Lett. 101, 171907 (2012)

30. Gu, X., Shaw, L., Gu, K., Toney, M. F. \& Bao, Z. The meniscus-guided deposition of semiconducting polymers. Nat. Commun. 9, 534 (2018).

31. Yao, Q. \& Wilkie, C. A. Thermal degradation of blends of polystyrene and poly(sodium 4-styrenesulfonate) and the copolymer, poly(styrene-co-sodium 4-styrenesulfonate). Polym. Degrad. Stab. 66, 379-384 (1999).

32. M’Bareck, C. O., Nquyen, Q. T., Metayer, M., Saiter, J. M. \& Garda, M. R. Poly (acrylic acid) and poly(sodium styrenesulfonate) compatibility by Fourier transform infrared and differential scanning calorimetry. Polymer 45, 4181-4187 (2004).

33. Nassyrov, D. et al. Vapour printing: patterning of the optical and electrical properties of organic semiconductors in one simple step. J. Mater. Chem. 22, 4519-4526 (2012).

34. Perevedentsev, A., Stavrinou, P. N., Bradley, D. D. C. \& Smith, P. Solutioncrystallization and related phenomena in 9,9-dialkyl-fluorene. I. Crystalline polymer-solvent compound formation for poly(9,9-dioctylfluorene). J. Polym. Sci. Part B Polym. Phys. 53, 1481-1491 (2015).

35. Perevedentsev, A., Stavrinou, P. N., Smith, P. \& Bradley, D. D. C. Solutioncrystallization and related phenomena in 9,9-dialkyl-fluorene. II. Influence of side-chain structure. J. Polym. Sci. Part B Polym. Phys. 53, 1492-1506 (2015).

36. Sundararajan, P. R. Small molecule self-assembly in polymer matrices. J. Polym. Sci. Part B Polym. Phys. 56, 451-478 (2018).

37. Luzzati, V. Structure cristalline de piasélénol, piazthiol et benzofurazane. Acta Cryst. 4, 193-200 (1951).

38. Michell, R. M. \& Müller, A. J. Confined crystallization of polymeric materials. Prog. Polym. Sci. 54-55, 183-213 (2017).

39. Jacobs, I. E. \& Moulé, A. J. Controlling molecular doping in organic semiconductors. Adv. Mater. 29, 1703063 (2017).

40. Lawson, J. R. \& Melen, R. L. Tris(pentafluorophenyl)borane and beyond: Modern advances in borylation chemistry. Inorg. Chem. 56, 8627-8643 (2017).

41. Pingel, P. et al. P-type poping of poly(3-hexylthiophene) with the strong Lewis acid tris(pentafluorophenyl)borane. Adv. Electron. Mater. 2, 1600204 (2016).

42. Panidi, J. et al. Remarkable enhancement of the hole mobility in several organic small-molecules, polymers, and small-molecule:polymer blend transistors by simple admixing of the Lewis acid p-dopant $\mathrm{B}\left(\mathrm{C}_{6} \mathrm{~F}_{5}\right)_{3}$. Adv. Sci. 5, 1700290 (2018).

43. Cochran, J. E. et al. Molecular interactions and ordering in electrically doped polymers: blends of PBTTT and F4TCNQ. Macromolecules 47, 6836-6846 (2014).

44. Patel, S. N. et al. Morphology controls the thermoelectric power factor of a doped semiconducting polymer. Sci. Adv. 3, el700434 (2017).

45. Zapata-Arteaga, O. et al. Closing the stability-performance gap in organic thermoelectrics by adjusting the partial to integer charge transfer ratio. Macromolecules 53, 609-620 (2020).
46. Vijayakumar, V. et al. Effect of alkyl side chain length on doping kinetics, thermopower, and charge transport properties in highly oriented F4TCNQdoped PBTTT Films. ACS Appl. Mater. Interfaces 11, 4942-4953 (2019).

47. Gao, J., Thomas, A. K., Johnson, R., Guo, H. \& Grey, J. K. Spatially resolving ordered and disordered conformers and photocurrent generation in intercalated conjugated polymer/fullerene blend solar cells. Chem. Mater. 26 4395-4404 (2014)

48. Francis, C. et al. Raman spectroscopy and microscopy of electrochemically and chemically doped high-mobility semiconducting polymers. J. Mater. Chem. C. 5, 6176-6184 (2017).

49. Yu, J., Abley, M., Yang, C. \& Holdcroft, S. Chemically amplified photolithography of a conjugated polymer. Chem. Commun. 15, 1503-1504 (1998).

50. Lee, C.-W., Seo, Y.-H. \& Lee, S.-H. A soluble polyaniline substituted with tBOC: Conducting patterns and doping. Macromolecules 37, 4070-4074 (2004).

51. Jacobs, I. E. et al. Direct-write optical patterning of P3HT films beyond the diffraction limit. Adv. Mater. 29, 1603221 (2017).

52. Bertho, S. et al. Influence of thermal ageing on the stability of polymer bulk heterojunction solar cells. Sol. Energy Mater. Sol. Cells 91, 385-389 (2007).

53. Müller, C. On the glass transition of polymer semiconductors and its impact on polymer solar cell stability. Chem. Mater. 27, 2740-2754 (2015).

54. Tasch, S. et al. Efficient red- and orange-light-emitting diodes realized by excitation energy transfer from blue-light-emitting conjugated polymers. Phys. Rev. B 56, 4479-4483 (1997).

55. Wolk, M. B., Lamansky, S. \& Tolbert, W. A. Progress in laser induced thermal imaging of OLEDs. SID Int. Symp. 39, 511-514 (2008).

56. Žemaitis, A. et al. Advanced laser scanning for highly-efficient ablation and ultrafast surface structuring: experiment and model. Sci. Rep. 8, 17376 (2018).

57. Pascual-San-José, E. et al. Towards photovoltaic windows: scalable fabrication of semitransparent modules based on non-fullerene acceptors via laserpatterning. J. Mater. Chem. A 8, 9882-9895 (2020).

58. Zhang, H., Poliks, M. D. \& Sammakia, B. A roll-to-roll photolithography process for establishing accurate multilayer registration on large area flexible films. J. Disp. Technol. 6, 571-578 (2010).

59. Wolk, M. B. et al. Laser thermal patterning of OLED materials. Proc. SPIE 5519, 12-23 (2004).

60. Malinowski, P. E. et al. High resolution photolithography for direct view active matrix organic light-emitting diode augmented reality displays. J. Soc. Inf. Disp. 26, 128-136 (2018).

61. Dörling, B. et al. Photoinduced p- to n-type switching in thermoelectric polymer-carbon nanotube composites. Adv. Mater. 28, 2782-2789 (2015).

62. Xu, Y. et al. Doping: a key enabler for organic transistors. Adv. Mater. 30, 1801830 (2018)

63. Khim, D. et al. Uniaxial alignment of conjugated polymer films for highperformance organic field-effect transistors. Adv. Mater. 30, 1705463 (2018)

64. Perevedentsev, A. et al. Assembly-induced bright-light emission from solution-processed platinum(II) inorganic polymers. ACS Omega 4, 10192-10204 (2019)

65. Kocher, C., Weder, C. \& Smith, P. Dichroic ultraviolet light filters. Appl. Opt. 42, 5684-5692 (2003).

66. Wigenius, J. A., Fransson, S., von Post, F. \& Inganäs, O. Protein biochips patterned by microcontact printing or by adsorption-soft lithography in two modes. Biointerphases 3, 75-82 (2008).

67. Ahmed, I. et al. An overview of smart packaging technologies for monitoring safety and quality of meat and meat products. Packag. Technol. Sci. 31, 449-471 (2018)

\section{Acknowledgements}

We are indebted to Enrique Pascual (ICMAB-CSIC) for providing insights into laser patterning and Lou Kiss (Middlesex University) for freely shared advice on the preparation of the graphics. We express our deepest gratitude to Paul Smith (ETH Zürich) for critically reviewing the manuscript. We thank Miquel Casademont-Viñas (ICMABCSIC) for invaluable contribution to exploring the limits of the method, and Agustín Mihi and Andrés Gómez (ICMAB-CSIC) for assistance with optical spectroscopy and AFM analysis. We also thank Daniel Kremer and Hans-Werner Schmidt (Universität Bayreuth) for their customary hospitality and assistance with DSC measurements. We further acknowledge financial support from the Spanish Ministry of Economy, Industry and Competitiveness through the "Severo Ochoa" Programme for Centers of Excellence in R\&D (SEV-2015-0496) and project reference PGC2018-095411-B-I00, as well as the European Research Council (ERC) under grant agreement number 648901.

\section{Author contributions}

A.P. and M.C.Q. conceived the project and designed the experiments. A.P. fabricated the samples, performed the experiments and analysed the data. A.P. and M.C.Q. wrote the manuscript. 


\section{Competing interests}

A patent application (EP20382514) has been filed based on the results reported herein. The authors declare no competing interests.

\section{Additional information}

Supplementary information is available for this paper at https://doi.org/10.1038/s41467020-17361-8

Correspondence and requests for materials should be addressed to A.P. or M.C.-Q.

Peer review information Nature Communications thanks the anonymous reviewer(s) for their contribution to the peer review of this work. Peer reviewer reports are available.

Reprints and permission information is available at http://www.nature.com/reprints
Publisher's note Springer Nature remains neutral with regard to jurisdictional claims in published maps and institutional affiliations.

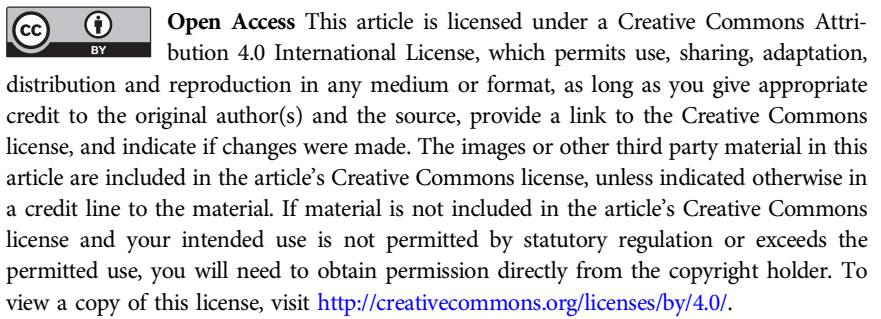

(C) The Author(s) 2020 